\title{
Leitorientierung Inklusion
}

\section{Eine Herausforderung für alle}

\author{
INGMAR STEINHART \\ Prof. Dr. phil. Ingmar Steinhart ist \\ Geschäftsführer im Stiftungsbe- \\ reich Bethel.regional der v. Bodel- \\ schwinghschen Stiftungen Bethel, \\ Direktor des Institutes für Sozialpsy- \\ chiatrie Mecklenburg-Vorpommern \\ An-Institut der Ernst-Moritz-Arndt- \\ Universität Greifswald, wissen- \\ schaftlicher Leiter der Modellprojek- \\ te des Landesverbandes Sozialpsy- \\ chiatrie Mecklenburg-Vorpommern \\ e. V. und Mitglied im Vorstand der \\ Aktion Psychisch Kranke e. V. \\ Internet http://http://www.bethel- \\ regional.de
}

\author{
Das Ziel einer "inklusiven Gesellschaft" \\ erfordert Anstrengungen von allen. Die sozialen \\ Leistungsträger und Leistungsanbieter sind dabei \\ fachlich wie leistungsrechtlich gefordert.
}

Die selbstverständliche Teilhabe von Menschen mit Behinderungen oder Psychiatrie-Erfahrungen an allen gesellschaftlichen Bereichen wird als Inklusion bezeichnet: Menschen mit Behinderungen sind wie alle anderen Menschen Bürgerinnen und Bürger uneingeschränkt, ungehindert, mit allen Rechten, aber auch mit Verpflichtungen.

Dabei beschreibt der Begriff der Inklusion kein statisches Ziel, sondern die Voraussetzung für generelle Teilhabe aller Menschen an gesellschaftlichen Möglichkeiten. Vor allem enthält der Begriff der Inklusion einen subjektorientierten sozialtherapeutischen bzw. sozial-pädagogischen und einen sozialpolitischen Auftrag: nämlich die Umgestaltung der Umwelt im Sinne einer Gesellschaft, die die Teilhabe aller ermöglicht, indem sie die Bürgerrechte aller respektiert und zu realisieren hilft. Und am Ende des Übergangs macht sich die Leitorientierung Inklusion selbst überflüssig: Mit der Überwindung der Exklusion benötigen wir auch keine Schärfung auf das Thema Inklusion, sondern wir können uns auf die »Teilhabe aller« konzentrieren.

Akzeptiert man für die nächste Dekade Inklusion als Auftrag insbesondere für das professionelle Hilfesystem, so werden viele aktuelle Angebotsformen für Hilfen zur Teilhabe ebenso infrage gestellt wie psychiatrische Behandlungsleistungen, denn ein solcher Auftrag führt über die bisherige Praxis der Integration hinaus.

Inklusion beinhaltet mehr als eine Normalisierung beeinträchtigten Le- bens durch Anpassung an sogenannte normale Lebensstandards: Inklusion ist dann noch lange nicht erreicht, wenn Menschen mit Behinderungen in einer Gemeinschaft mit anderen leben oder wenn eine ausreichende Anzahl an speziellen Bildungs-, Beschäftigungs- und Freizeitangeboten für diesen Personenkreis zur Verfügung steht. Vielmehr geht es um die Ermöglichung eines individuell gestalteten Lebens und um die Einbeziehung von Menschen, die anders erscheinen, in die normalen Alltagsund Lebensvollzüge einer Gesellschaft - eingeschlossen die Pflicht für diese, ihre eigenen Fähigkeiten und Ressourcen einzubringen.

Wesentliches Grundmoment ist hierbei die selbstverständliche Begegnung von Menschen mit und ohne Psychiatrie-Erfahrung oder Behinderungen insbesondere beim Wohnen, bei der Arbeit und in der Freizeit. Neben dieser "Öffnung « der Gesellschaft gilt es auch weiterhin, »Schutzräume « oder »Asyle « zu erhalten oder neu zu definieren - sofern die Menschen dieses wollen.

Eine Umsetzung erfordert vor allem eine neue Professionalität. Damit tritt Inklusion (fast) allen auf die Füße: Mit einem Perspektivwechsel von der Betreuung und Bewahrung über die Integration zur inklusiven Gesellschaft und mit einem Umsetzungskonzept im Lebensfeld und in den Sozialräumen statt in Institutionen ist gelebte Inklusion in der Praxis stets mit starker eigener Veränderung verbunden.

Betroffen sind gleichermaßen die Systeme der öffentlichen Verwaltungen, der Leistungsträger, der Wohlfahrtsver- 


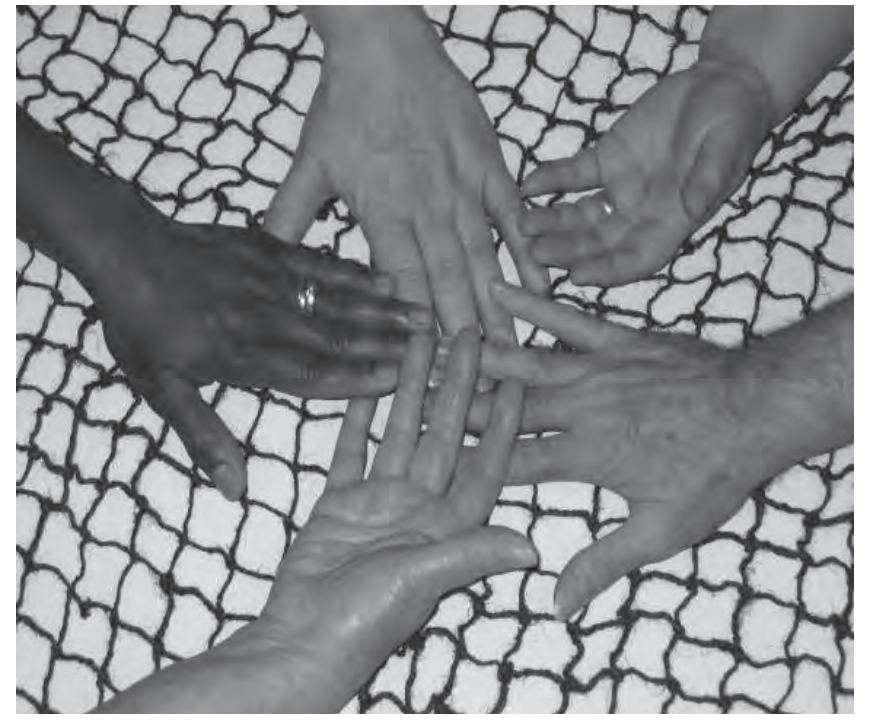

Folgt man dem Ansatz der Inklusion und den daraus abzuleitenden Schritten für die Leistungserbringung konsequent, so werden letztendlich alle Leistungen ambulant bzw. es entfällt die Unterscheidung zwischen ambulant und stationär. Das bedeutet, neben der Verfügbarkeit von individuellem Wohnraum und einem individuellen Arbeits- bzw. Beschäftigungsplatz möglichst an den Orten, wo auch nicht behinderte Bürgerinnen und Bürger wohnen und arbeiten, kommt es darauf an, ausgehend vom individuellen Hilfebedarf entweder den Sozialraum im Sinne der Inklusion zu stützen und zu stärken und/oder den Klienten selbst. Dafür sind zwei Leistungstypen ausreichend:
1. Unterstütztes Leben und Arbeit in der Gemeinde (prospektiv, zeitbasiert, fallspezifisch)

\section{Stärkung des Sozialraums (pauschaliert, fallunspezifisch)}

Die Forderung, dass alle Leistungen im Grundsatz "ambulant" sind und dass Person und Sozialraum in der Finanzierung gleichrangig nebeneinanderstehen, beinhaltet nicht nur leistungsrechtlich ein Umdenken, sondern verändert auch die allgemeine Haushaltsystematik der Kommunen, die im Allgemeinen die Stärkung des Sozialraums als "freiwillige" Leistungen betrachten.

Sofern sich in der bundesweiten Zuständigkeit nichts ändert, müssten Leistungen zur Teilhabe im Sinne einer "Normalisierung der Finanzierung " konsequenterweise von den Ländern an die Kommunen im Rahmen des Finanzausgleiches transferiert werden und nicht mehr über "Sonderformen" wie z. B. "Sozialhilfefinanzierungsgesetze«. Es sei hier die steile These gewagt, dass sich perspektivisch unter den Aspekten der Primär-, Sekundär- und Tertiärprävention die sogenannten direkten (personenbezogenen) Leistungen im Verhältnis zu den indirekten sozialraumbezogenen Leistungen auf ein Verhältnis von 50:50 "einpendeln« werden. Und das wird/muss sich auch in der Finanzierung und ihrer Systematik widerspiegeln.

Ingmar Steinhart

Quelle: Ingmar Steinhart, Umsteuerung zu flexiblen Hilfen - Nehmen wir Personen- und Lebensweltbezug endlich ernst! Internet http://www.apk-ev.de/publikationen/Band_35.pdf bände und der Leistungsanbieter - nicht zuletzt der psychiatrischen Kliniken und Abteilungen, und letztendlich jeder Einzelne, der in diesen Systemen mitarbeitet. Eine Aufbruchstimmung lässt sich überall da erkennen, wo dieser Auftrag angenommen und als Wert in das jeweilige System integriert wird.

\section{Erste Herausforderung: Barrierefreiheit}

Barrierefreiheit beginnt zunächst in den Köpfen aller Beteiligten, muss aber die Herzen erreichen und kann am ehesten befördert werden durch eine Beteiligung von Menschen mit Psychiatrie-Erfahrung bzw. Behinderung insbesondere an Entscheidungsprozessen.

Ein Leben in der Normalität des örtlichen Sozialraums setzt die »umfassende Barrierefreiheit « von (öffentlichen) Diensten im Sinne der Zugänglichkeit und Nutzbarkeit voraus (z. B. Zugang zu Wohnraum und Arbeit, die Erreichbarkeit der persönlichen Hilfen, wie z. B. durch ambulante und mobile Rund-um-die-Uhr-Dienste, durch die
Senkung der Zugangsschwellen von Hilfen etc.).

\section{Zweite Herausforderung: Gemeinwesen}

Das Gemeinwesen muss bereit und dazu fähig sein, alle Menschen, auch diejenigen, die vermeintlich anders sind, anzunehmen und aufzunehmen. Um dieses Ziel, also die Beendigung von Exklusion, irgendwann einmal zu erreichen, muss vorab eine Stärkung der Zivilgesellschaft über ein gezieltes Befähigungskonzept des Gemeinwesens erfolgen: Unter anderem werden Erfahrungen aus der direkten Perspektive von Menschen mit Behinderung in den üblichen Beratungsprozessen der Bürgerschaft, wie beispielsweise im Parlament, in Ausschüssen, in Haushalts- oder Bebauungsplänen zum Thema. Außerparlamentarisch könnten die Bürgerinnen und Bürger über Bürgerplattformen oder vergleichbare Methoden befähigt werden, für ihre eigenen Belange einzutreten und ihre Lebensbedingungen auszugestalten.

\section{Dritte Herausforderung: Netzwerke}

Da die Gesellschaft selbst inklusiv(er) werden soll, benötigen Menschen mit Behinderungen oder mit PsychiatrieErfahrungen differenzierte Netzwerke, die sie bei ihrer gleichberechtigten Teilhabe unterstützen: nicht-professionelle, semiprofessionelle oder professionelle. Es entstehen neue Professionen, wie beispielsweise der Community-Networker, der über seine indirekte Brückenarbeit im Gemeinwesen dazu beitragen soll, Exklusion zu verhindern. Gemeinwesenarbeit und Know-how über indirekte Interventionen bei der Knüpfung von Netzwerken sind hier als Kernkompetenzen gefordert - die Beteiligung von Erfahrenen in der praktischen Netzwerkarbeit und in der Ausbildung der Profis ist hierbei letztlich unabdingbar.

Auch der weitere Aus- und Aufbau trialogischer und quadrologischer Netzwerke mit Erfahrenen, Profis und Angehörigen, Bürgern bzw. wesentlich Beteiligten unterstützt den inklusiven Entwicklungsprozess. 


\section{Vierte Herausforderung: Teilhabe}

Menschen mit Behinderungen wollen nicht nur Empfänger von Hilfen und Leistungen sein, sondern sie wollen auch selbst geben. Wesentliches Ziel ist, dass Menschen mit Psychiatrie-Erfahrungen bzw. Behinderungen ihre Rolle des Hilfe-Empfangenden verlassen und in die Rolle des (z. B. ehrenamtlich) Gebenden einsteigen. Sie gewinnen hierbei aktive Teilhabe und Normalität.

\section{Die Wohlfahrtspflege neu denken}

Die professionellen Hilfesysteme wie u. a. die Angebote der Freien Wohlfahrtspflege werden sich dem Leitgedanken der Inklusion unterordnen, die »inklusive Gesellschaft" als Leitidee im professionellen Alltag befördern und sich an die Spitze der Bewegung setzen (müssen).

Die neuen psychosozialen Dienstleistungen zeichnen sich dadurch aus, dass sie sich an den persönlichen Voraussetzungen eines Menschen, den jeweiligen Anforderungen in seiner Lebenswelt und sich daraus ergebenden hemmenden Faktoren der Partizipation orientieren. Die sozialen Dienstleister sind damit aufgefordert, zu einer ressourcenund autonomiefördernden Lebensgestaltung beizutragen. Statt reflexartig mit institutionalisierten oder vorgefertigten Lösungen zu reagieren, könnte die professionelle Herausforderung darin bestehen, zuzuhören und nach Antworten zu suchen. Antworten zu finden auf die Fragen, die Menschen mit speziellen Erfahrungen stellen, die in einer Gemeinde ihre persönlichen Zukunftsperspektiven entwickeln möchten. Inwieweit die Beantwortung mit (nicht-) professionellen Mitteln gelingt, könnte eine Messlatte für den Inklusionsfortschritt bedeuten.

Partnerschaften zwischen der Wohlfahrtspflege, Institutionen, öffentlichen Dienstleistungen, gemeindlichem Leben, bürgerschaftlichem Engagement und privaten Hilfen müssen neu entstehen und zu einem konstruktiven Zusammenspiel gebracht werden. Die Wohlfahrtspflege ist hier besonders gefordert für die Entwicklung einer inklusiven Gesellschaft Vorbildfunktion zu übernehmen - und unterwegs niemanden $\mathrm{zu}$ verlieren: weder die eigenen Mitarbeitenden - unabhängig vom Schweregrad des erforderlichen Perspektivwechsels - noch Menschen mit (besonderen) Erfahrungen.

\section{Die psychiatrische Klinik und Behandlung neu denken}

Nicht nur neue personen- und lebensfeldorientierte Therapieansätze wie beispielsweise aus Skandinavien das "Need Adapted Treatment « oder der »Open Dialogue«, sondern vor allem die übergreifende Leitidee Inklusion sollten die SGB-V-Psychiatrie einschließlich der Finanziers, der Krankenkassen, dahin bewegen, die Therapiekonzepte und Angebotsstrukturen zu überdenken. Auch sie sollten sich an den persönlichen Voraussetzungen eines Menschen, den jeweiligen Anforderungen in seiner Lebenswelt und den sich daraus ergebenden Erschwernissen der Partizipation orientieren.

Eine der wirksamsten psychiatrischen Interventionsmethoden ist die Arbeit mit Angehörigen oder wesent- mitgestalten zu können. Sie müssen im Rahmen gezielter Organisations- und Personalentwicklung in dieser Aufgabe unterstützt werden.

- Basis-Mitarbeitende: Sie müssen den im Sinne von Inklusion erforderlichen Perspektivwechsel in der von ihnen zu erbringenden Arbeitsleistung als Wert mittragen, die Prozesse mitgestalten und die Umsetzung mitmachen.

- Verwaltung, Krankenhaus(leitung): Auch die Mitarbeiter und Mitarbeiterinnen der Verwaltung (der Kommunen wie der Leistungsanbieter inklusive Krankenhäuser) müssen die Wertediskussion und den Paradigmenwechsel nicht nur mittragen, sondern direkt unterstützen. Allein die Umstellung von »immobiliengestützten « institutionellen Angeboten hin zu ambulanten »nicht an Immobilien gebundenen «, also gemeinwesenintegrierten Angeboten, ist eine große Herausforderung.

\section{"Die Wohlfahrtspflege darf auf dem Weg zu einer inklusiven Gesellschaft niemand verlieren, auch nicht die eigenen Mitarbeiterinnen und Mitarbeiter"}

lichen Bezugspersonen. Interventionen mit der Lebenswelt (Angehörigenarbeit) und im Lebensfeld (z. B. ambulante Krisenintervention, »Home-Treatment «) könnten zum Kern der psychiatrischen Behandlungsmaßnahmen werden; die klassische Klinik würde nur noch als »Rückzugsraum« dienen. Ein »inklusives Behandlungssetting «, einschließlich der damit verbundenen präventiven Bemühungen im Sinne der Befähigung des Gemeinwesens, hätte am Ende die Vision einer "Aufhebung der klassischen Klinik« zum Ergebnis.

\section{Profis werden inklusionsfit}

Alle an dem Prozess beteiligten Profis (Anbieter, Leistungsträger, Kommunalverwaltung) müssen sich neu orientieren, um den Perspektivwechsel aktiv

\section{Leistungsträger müssen Ressourceneinsatz neu denken}

Zum Beispiel könnte ein »TeilhabeWeiterentwicklungsgesetz « über einen Zeitraum von fünf bis zehn Jahren zusätzliche Finanzmittel wie einen "Inklusions-Euro « in jeder Fachleistungsstunde oder jeder Maßnahmepauschale etc. bereitstellen - verbunden mit einem Anreiz- und Belohnungssystem zur Systemtransformation. Sektorübergreifende SGB-V-Regionalbudgets der Krankenkassen können personenorientierte Behandlungsleistungen im Lebensfeld ermöglichen und die Anreize weg von der »Fallzahlerhöhung « hin zu "mehr an Teilhabe « setzen. Strukturverändernde (!) Finanzierungsmodelle und Finanzierungsanreize vor allem im SGB V-Bereich sind jetzt zu etablieren. 


\section{Kommunale Konversions- strukturen aufbauen}

Inklusion ist eine Entwicklung, die im Gemeinwesen, also auf kommunaler Ebene bzw. im Bereich der jeweiligen Gebietskörperschaften, stattfindet. Letzteren kommt daher eine besondere Verantwortung im Rahmen des Transformationsprozesses zu. Die Installation einer örtlichen Teilhabeplanung könnte die Perspektive für eine Verwaltung "gangbar machen". Strukturelemente könnten sein:

- Regionale Inklusionsverwirklichungskonferenzen, die unter Beteiligung aller Akteure im Gemeinwesen die Gesamtentwicklung beobachten und für einen strukturierten Entwicklungsprozess in Richtung eines »inklusiv(er)en Gemeinwesens « Sorge tragen.

- Regionale Teilhabeverbünde im Sinne einer regionalen inklusiven Management-Gesellschaft könnten für die Abstimmung aller beteiligten Akteure und der Steuerung der Finanzmittel sorgen.
Entwicklung einer inklusiven Finanzierungssystematik, die fallunspezifische inklusive präventiver sowie fallspezifische Leistungen enthält und den regionalen Budget-Gedanken inklusive des Persönlichen Budgets weiterentwickelt

\section{Individuelle Teilhabe verwirklichen}

Um den Weg zu einer inklusiven Gesellschaft auf der individuellen Ebene für Menschen mit und ohne Behinderungen zu schultern, müssen die bestehenden Steuerungsmodelle (Clearingverfahren, Hilfeplanung, Teilhabeplanung, Gutachten durch den Medizinischen Dienst der Krankenkassen etc.) so weiterentwickelt werden, dass sie gleichberechtigte Teilhabe praktizieren und eine Begegnung auf Augenhöhe zulassen.

\section{Vollversorgung realisieren oder: Inklusion ist ein Konzept für alle}

Die Zeichen gesellschaftlicher Entwicklungen sind zurzeit eher auf Exklusion gerichtet; gleichwohl entwickelt sich eine Bewegung in Richtung der Stärkung des gesamtgesellschaftlichen Systems zur Überwindung dieses Trends zur Exklusion und zur Befähigung der Gesamtgesellschaft zu mehr Inklusion. Hier gilt es, sich an den alten sozialpsychiatrischen Grundwert der regionalen Vollversorgung, also an das regionale Exklusionsverbot (!), zu erinnern.

Besonders gefährdet ausgeschlossen $\mathrm{zu}$ werden sind diejenigen, die einen besonders hohen Unterstützungsbedarf haben oder besonders unattraktiv sind, wie Menschen mit schwersten chronischen Verläufen, Menschen mit sogenanntem herausforderndem Verhalten, Menschen, die aus der forensischen Psychiatrie entlassen werden und viele andere mehr. Nur wenn es gelingt, gerade für diesen Personenkreis Fortschritte in der Teilhabe auch in der Praxis zu verwirklichen, kann letztlich von einem gelingenden Inklusionsprozess und von einem Inklusionsfortschritt ausgegangen werden.

\section{Aktuell: SGB II-Reform berücksichtigt.}

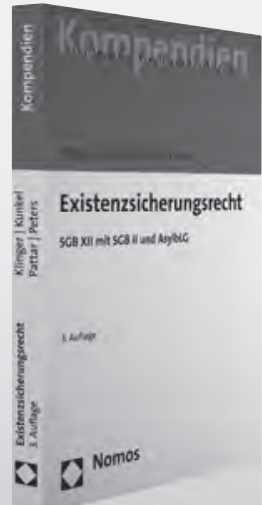

Das Werk ist eine systematische Darstellung des Existenzsicherungsrechts in SGB I, II, X, XII und des Asylbewerberleistungsgesetzes. Außerdem enthält es die Hilfen in unterschiedlichen Lebenslagen nach dem SGB XII. Mit Prüfschemata und Schaubildern will es speziell Studierenden, aber auch Praktikern Arbeitshilfe leisten.

\section{Existenzsicherungsrecht}

SGB XII mit SGB II und AsylbLG

Von Prof. Roland Klinger, Prof. Peter-Christian Kunkel,

Prof. Dr. Andreas Kurt Pattar und Ri'in Karen Peters

3. Auflage 2012, 499 S., brosch., 28,- $€$

ISBN 978-3-8329-6265-4

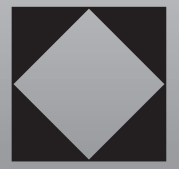

Nomos 